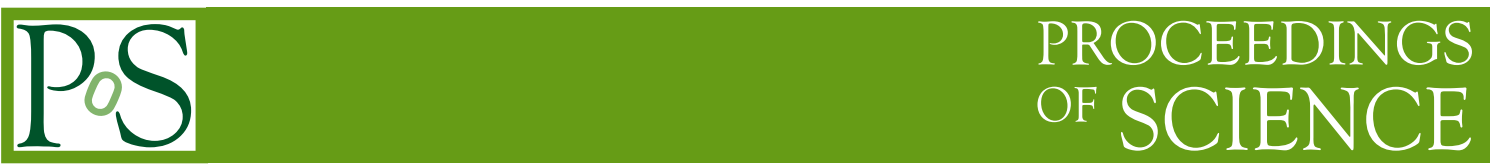

\title{
Results from the Pierre Auger Observatory
}

\author{
Vitor de Souza* for the Pierre Auger Collaboration \\ Instituto de Física de São Carlos - Universidade de São Paulo \\ E-mail: vitoreifsc.usp.br \\ Full author list: http://www.auger.org/archive/authors_2010_08.html
}

\begin{abstract}
The Pierre Auger Observatory is running continuously and taking good quality data. In this article we review some of the main results from the Observatory regarding the cosmic ray energy spectrum, the primary particle composition and the arrival directions. By measuring the energy spectrum for energies above $10^{19} \mathrm{eV}$ with unprecedented significance the Pierre Auger Observatory has undoubtly determined a GZK-like suppression in the flux of particles reaching Earth. An anisotropy in the arrival direction of the particles has been measured for energies above $5.5 \times 10^{19}$ $\mathrm{eV}$ including an excess in the direction of the nearest radio loud active galaxy, Centaurus A. An evolution of the average mass of primary particle has also been determined by measuring the depth at which showers reach the maximum number of particles. This data shows the evolution of the average mass from light to heavier when compared to hadronic interaction models extrapolated from data taken by particle physics experiments. These three main results measured by the Pierre Auger Observatory define the actual cosmic ray puzzle which scientists around the world are working on in order to understand and elaborate a coherent picture. We review these results here and point to future possibilities.
\end{abstract}

4th School on Cosmic Rays and Astrophysics,

August 25-September 04, 2010

Sao Paulo Brazil

${ }^{*}$ Speaker. 


\section{Introduction}

The Pierre Auger Observatory has been taking data since 2004 [1] in its hybrid mode. The Auger Observatory is the first experiment to combine the two main techniques for detection of extensive air showers: fluorescence light telescopes and water Cherenkov tanks as surface detectors. Figure 1 shows the arrangement of tanks and the fields of view of the telescopes.

The surface detector consists of 1600 water tanks that measure Cherenkov light produced by the passage of particles in the water. They are arranged in a regular grid with $1.5 \mathrm{~km}$ spacing. Each tanks is equipped with three photomultipliers and are powered by a solar panel system and batteries. The data is sent from each tank to a central acquisition system through a wireless connection thus making each tank a stand-alone detector.

Four fluorescent light detectors are fully operational at the Southern site of the Pierre Auger Observatory. In each of the four detectors there are six telescopes in operation. Each telescope covers $30^{\circ}$ azimuth and $30^{\circ}$ in elevation. They are equipped with Schmidt optics consisting of a segmented mirror of $11 \mathrm{~m}^{2}$, with radius of curvature of $3.4 \mathrm{~m}$ and a $2.2 \mathrm{~m}$ diameter diaphragm. The opening of the telescope is covered by a filter that selects the wavelength range corresponding to the emission of fluorescent light by nitrogen molecules in air (300 - $400 \mathrm{~nm})$. After going through this sophisticated experimental arrangement, light produced by air shower is captured by a set of 440 photomultipliers.

The use of both techniques simultaneously makes the Pierre Auger Observatory a hybrid de-

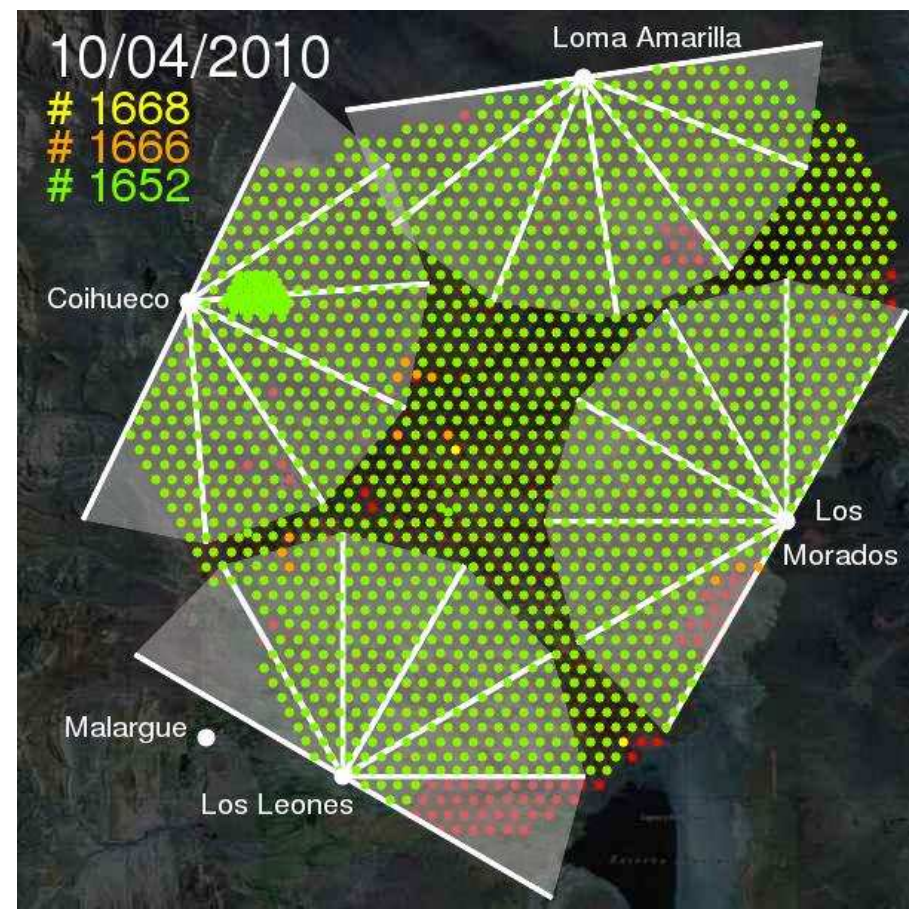

Figure 1: Pierre Auger Observatory Map. The points show the locations of the surface detectors. In red are the planned positions, in yellow detectors installed, in orange detectors filled with water and in green those that have already detected at least one event. The white line represents the fields of view of each fluorescence telescopes. 
tector resulting in a reduction of systematic errors and allowing cross-calibration between the different techniques. In particular, the combination of a well defined collection area offered by the surface detectors and a calorimetric energy measurement done by the fluorescence telescopes provides a unique detection ability to the Pierre Auger Observatory which has resulted in important discoveries [2-4] that are going to be reviewed in the next sections.

\section{Energy Spectrum}

The energy spectrum of cosmic rays with energy above $10^{19} \mathrm{eV}$ has been a controversy since the detection of the first ultra high energy (UHE) particle measured in Volcano Ranch [5]. The interactions of such a particle with energy above $5 \times 10^{19} \mathrm{eV}$ with the cosmic microwave background (CMB) imply that most of the flux above this energy should come from sources closer than $200 \mathrm{Mpc}$. This argument presented by Greisen [6], Zatsepin and Kuzmin [7] is known as the GZK effect.

Two experiments AGASA [8] and HiRes [9] using different techniques, scintillators as surface detectors and fluorescent telescopes, respectively, have measured, with a limited number of events, discrepant spectra. AGASA showed a hint of a continuous flux for energies above $5 \times 10^{19} \mathrm{eV}$ while HiRes measured a hint of a break in the flux around the same energy. Many suppositions about the detection techniques and different acceleration mechanisms have been proposed based on these measurements. The controversy was set by the large uncertainty in the determination of the flux due to a small, in case of AGASA, and not well defined, in case of HiRes, collection area.

In 2008, the Pierre Auger Observatory [4] resolved this controversy by determining the energy spectrum with very good energy resolution and well defined collection area. Figure 2 shows the measured energy spectrum. A break in the energy spectrum was found at $4 \times 10^{19} \mathrm{eV}$. If a powerlaw function in energy $\left(J \propto E^{-\gamma}\right)$ is fitted, the spectral index $(\gamma)$ is $2.69 \pm 0.02($ stat $) \pm 0.06$ (syst) below the break and $4.2 \pm 0.4$ (stat) \pm 0.06 (syst) above the break. Using these numbers, the departure from a continuous spectrum along the break point can be ruled out with a 5 sigma significance.

\section{Arrival Directions}

The arrival directions of cosmic ray particles provide important information on the locations of their sources. If the GZK effect takes place as expected, the source should be near enough so that we could point backwards and study individual sources. That also depends on the charge of the particle and on the magnetic fields as explained below.

Mechanisms that accelerated particles to the highest energies require special astrophysical environments. From one side, different classes of objects have been proposed as possible sources: active galactic nuclei (AGN), radio galaxy lobes and sources of gamma-ray bursts. On the other hand, more exotic mechanisms based on Z bursts, Super Heavy Dark Matter (SHDM) decays and topological defects have also been proposed. This exotic class of mechanisms has been named topdown because high energetic objects would interact/decay and produce the cosmic ray particles observed on Earth. 


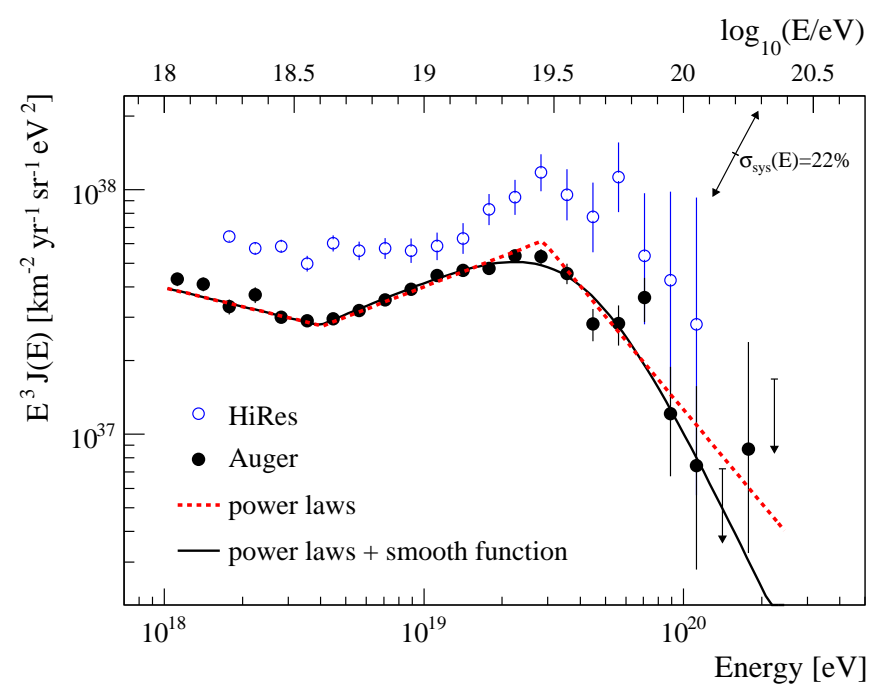

Figure 2: Energy spectrum measured by the Pierre Auger Observatory [4].

The two classes of mechanisms generate a completely different arrival direction pattern. Traditional sources are anisotropic in the nearby Universe while exotic mechanisms would produce an isotropic flux of cosmic particle arriving on Earth.

In 2007, the Pierre Auger Collaboration published a paper [2] in which the arrival directions of the highest energy particles correlated with a specific catalog of AGNs [10] showing therefore for the first time that these particles were generated in sources that are not distributed isotropically in the sky.

Figure 3 shows the directions of the highest cosmic rays compared with AGNs directions [11]. Figure 4 shows the evolution of the degree of correlation as a function of the number of measured events. This plot shows how the correlation probability departs from the expected in case of isotropy. It was calculated every time we measured a new event above $55 \mathrm{EeV}$. This energy threshold has been chosen as the one which maximized the correlation following the explanation in reference [12]. The color bars in figure 4 show respectively in red, blue and gray the $68 \%, 95 \%$ and $99.7 \%$ departure from the data. The dashed line shows the corresponding expected isotropy value.

\section{Composition}

The last important piece of information we can use in order to understand the production of highly energetic particle is the mass abundance. This is important because each acceleration mechanism produces a different abundance of elements ranging from proton to iron nuclei. The charge of the cosmic ray is also important in order to trace back the origin of the particle.

Cosmic particles are deviated in the galactic and extragalactic magnetic fields by amounts proportional to their charges. If the particles which arrive on Earth have a low charge they may point back to their source. However if the charge of the particle is larger, closer to iron, their directions could be greatly deviated and the pointing information erased. 


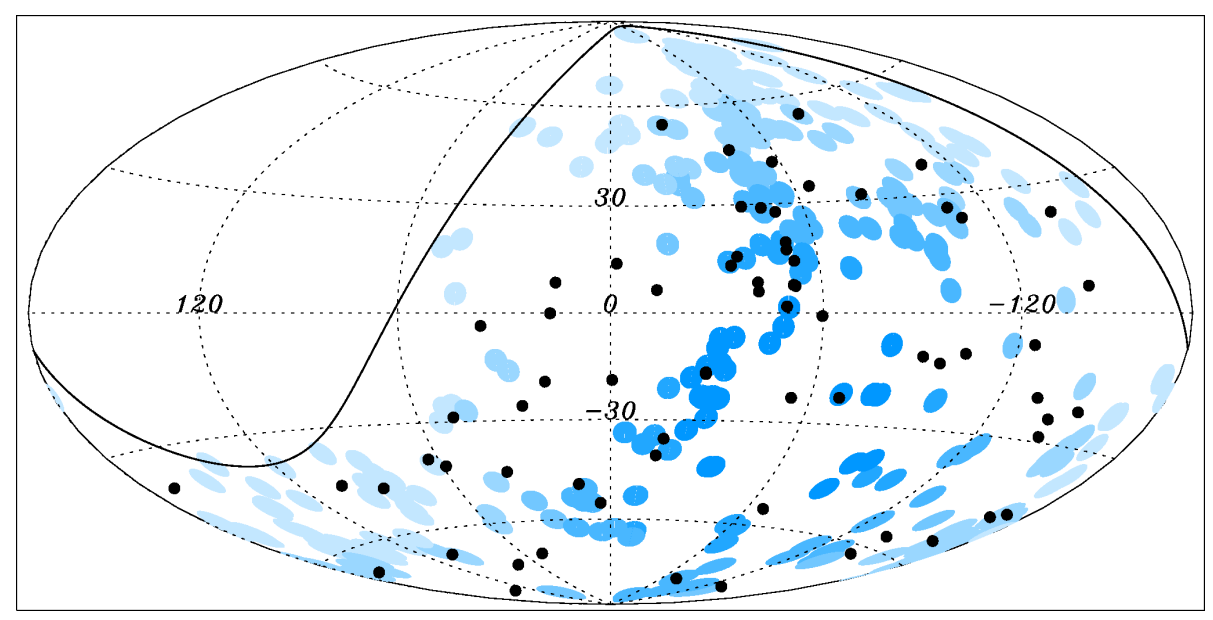

Figure 3: The 69 arrival directions of CRs with energy $E \geq 55 \mathrm{EeV}$ detected by the Pierre Auger Observatory up to 31 December 2009 are plotted as black dots in an Aitoff-Hammer projection of the sky in galactic coordinates. The solid line represents the field of view of the Southern Observatory for zenith angles smaller than $60^{\circ}$. Blue circles of radius $3.1^{\circ}$ are centred at the positions of the 318 AGNs in the VCV catalog that lie within $75 \mathrm{Mpc}$ and that are within the field of view of the Observatory. Darker blue indicates larger relative exposure. The exposure-weighted fraction of the sky covered by the blue circles is $21 \%$ [11].

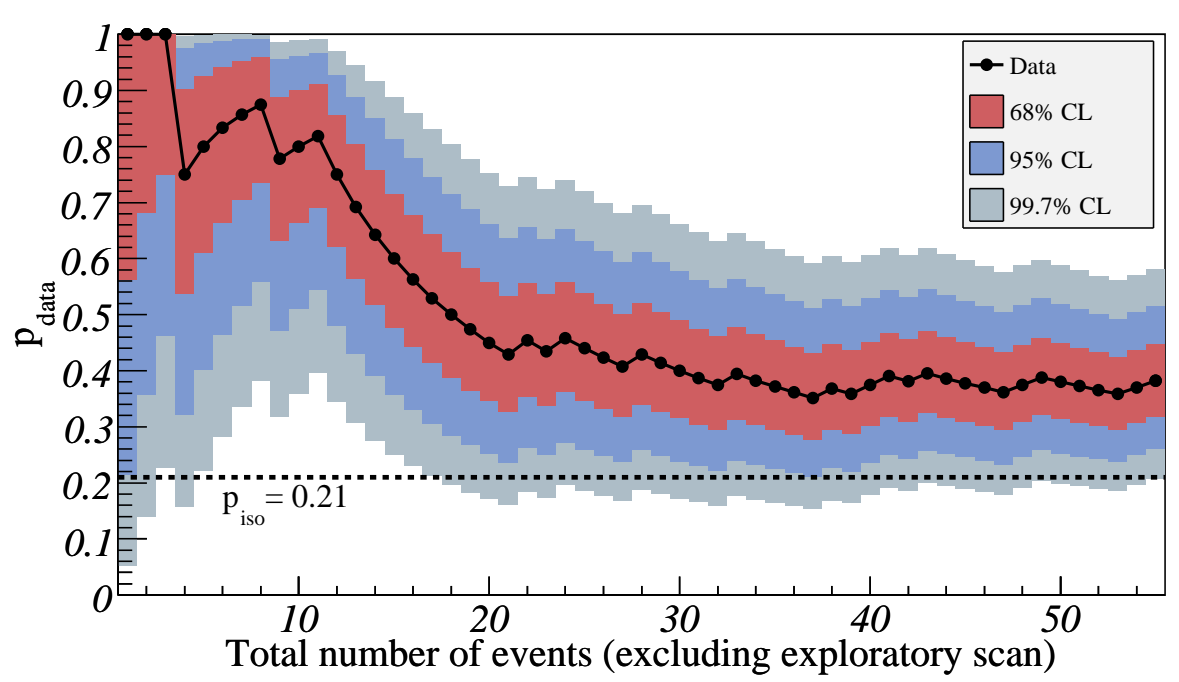

Figure 4: The most likely value of the degree of correlation $p_{\text {data }}=k / N$ is plotted with black dots as a function of the total number of time-ordered events (excluding those in period I). The 68\%, 95\% and $99.7 \%$ confidence level intervals around the most likely value are shaded. The horizontal dashed line shows the isotropic value $p_{\text {iso }}=0.21$. The current estimate of the signal is $\left(0.38_{-0.06}^{+0.07}\right)[11]$. 

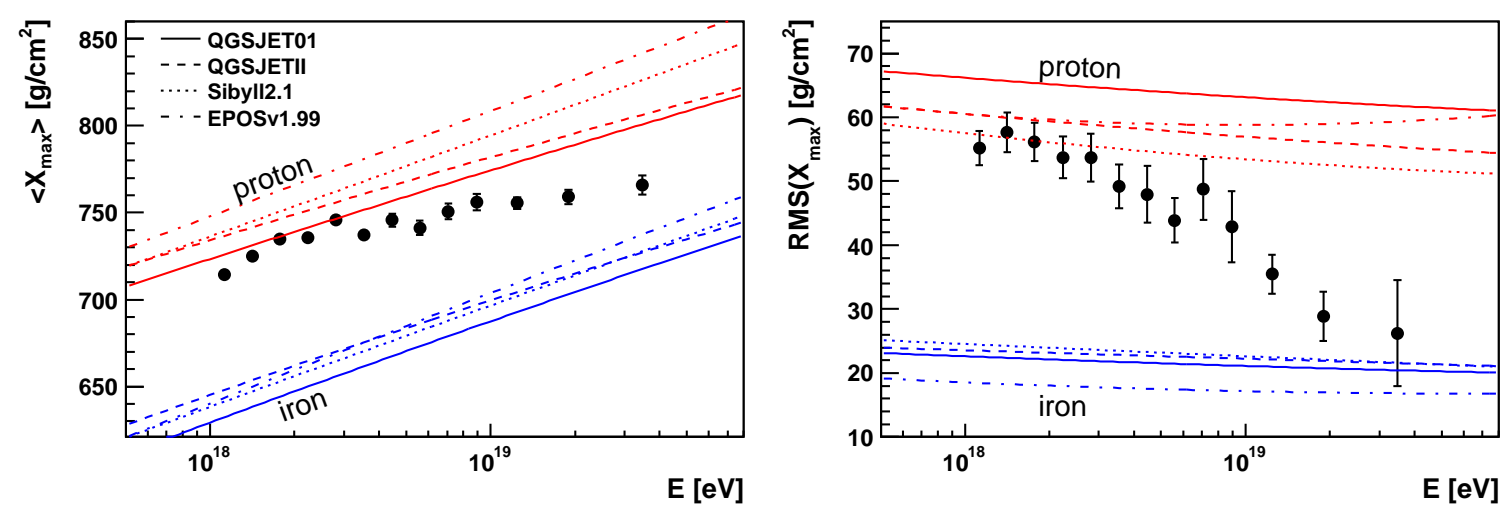

Figure 5: Figure a) on the left shows the evolution of $\left\langle X_{\max }\right\rangle$ and figure $b$ ) on the right shows the evolution of $\operatorname{RMS}\left(X_{\max }\right)$ as a function of energy [3] .

Currently, composition studies have been published using the data measured by fluorescence telescopes. Fluorescence telescopes are sensitive to the primary composition by measuring the depth at which the shower has the maximum number of particles $\left(X_{\max }\right)$. However, due to the large fluctuations in the shower development, plus the limited statistics and the uncertainties in the hadronic interaction models [13], the primary particle identification is very difficult and not possible on an event-by-event basis.

The Pierre Auger Observatory has published [3] the evolution of the mean $X_{\max }$ and of its fluctuation as a function of energy. Figure 5 shows the results in comparison with the predicted values for proton and iron primaries. A unique interpretation of these results is not independent of the hadronic interaction models at the highest energies. If the low energy interaction properties (cross sections and inelasticity) can be directly extrapolated to the highest energies, as considered in the models shown in figures $5 \mathrm{a}$ and $\mathrm{b}$, our data can be interpreted as a change in the cosmic ray composition starting light for energies around $10^{18} \mathrm{eV}$ and moving towards heavier primaries for energies around $10^{19} \mathrm{eV}$. However if the hadronic interaction properties change in this energy range the conclusion could be different.

Regarding photons and neutrinos the Pierre Auger Observatory has been also able to set very restrictive limits. Figure $6 a$ and $b$ show the limits imposed by our data.

\section{Final Remarks}

The Pierre Auger Observatory has set a new standard in high energy cosmic ray physics. The quality of the data, the careful analysis and the relatively large statistics has taken this research field to a quantitative era of high energy astroparticle physics. Hints and uncertainties from the past have been definitely confirmed or refuted and the parameter space for models has been firmly restricted. New discoveries have been made and the data shows hints that many more could be seen in the near future.

The high energy cosmic ray picture shows a) a suppression of the flux for energies above $4 \times 10^{19} \mathrm{eV}$ compatible with the GZK effect; b) an anisotropic arrival distribution for energies 

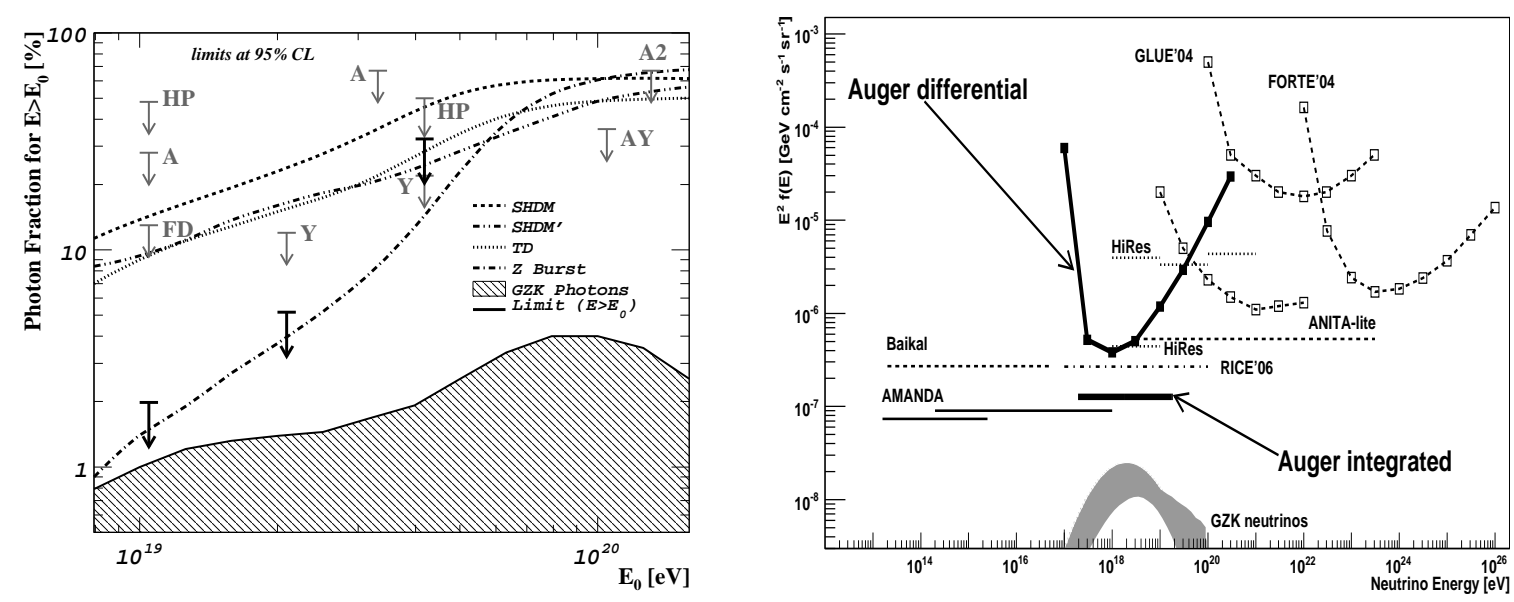

Figure 6: Figure a) on the left shows the Auger photon fraction (solid upper limits) in the integral cosmic-ray flux compared to other experiments and theoretical predictions from top-down models and for GZK photons. For references see [14] b) on the right shows the $90 \%$ CL for each flavor of diffuse UHE neutrino fluxes assuming a proportion of flavors of 1:1:1 due to neutrino oscillations. It is also shown other experiments and theoretical predictions from top-down models and for GZK photons [15].

above $5.5 \times 10^{19} \mathrm{eV}$; c) a conditional evolution from light $\left(10^{18} \mathrm{eV}\right)$ to heavy primaries $\left(10^{19} \mathrm{eV}\right)$; d) strict neutrino and photon flux limits.

Some important challenges are imposed by this picture. If heavy primaries are more abundant at energies around $10^{19} \mathrm{eV}$ they would be strongly deviated by magnetic fields and the correlation with the source(s) could be erased. However, the data shows that in an energy range slightly above the $X_{\max }$ measurements, above $5.5 \times 10^{19} \mathrm{eV}$, an anisotropic sky is seen. Could there be an abrupt mass transition between $10^{19} \mathrm{eV}$ and $5.5 \times 10^{19} \mathrm{eV}$ ? Or is the change in $\left\langle X_{\max }\right\rangle$ caused by new physics in particle interactions?

The sources of these particles are still to be discovered. Important information has been delivered by the anisotropy measurements. However we have not been able to identify the class of objects responsible for the majority of the flux. A recent publication [11] shows an excess around the direction of Cen A. In a circle within $18^{\circ}$ around Cen A 13 events were measured while 3.2 would be expected from an isotropic distribution. Figure 7 shows the cumulative number of events as a function of the angular distance from Cen A. An excess around $18^{\circ}$ is clearly seen.

Classified as an FRI radio-active galaxy, Cen A is a complex object and considered a prototype of this class of active galaxies. Two bipolar jets are observed at length emerging from the radio nucleus of this galaxy with angular dimensions of $8^{\circ} \times 4^{\circ}$, extending for millions of light years in the intergalactic space. The structure of these jets is also quite rich, with sub-jets and bright knots along their length formed by magnetized shocks where it is believed that cosmic rays are accelerated. Recent estimates consider a central black hole with mass of about $5 \times 10^{7}$ solar masses [16] and a kinetic power of the jets of the order of $10^{43} \mathrm{erg} / \mathrm{s}$ [17].

Given the complex and extended structure of Cen A, several models for particle acceleration above $10^{18} \mathrm{eV}$ have been proposed: a) the acceleration on the outskirts of the central black hole; $\mathrm{b}$ ) direct acceleration in the internal knots along the jets; c) re-acceleration of the particles, originally 


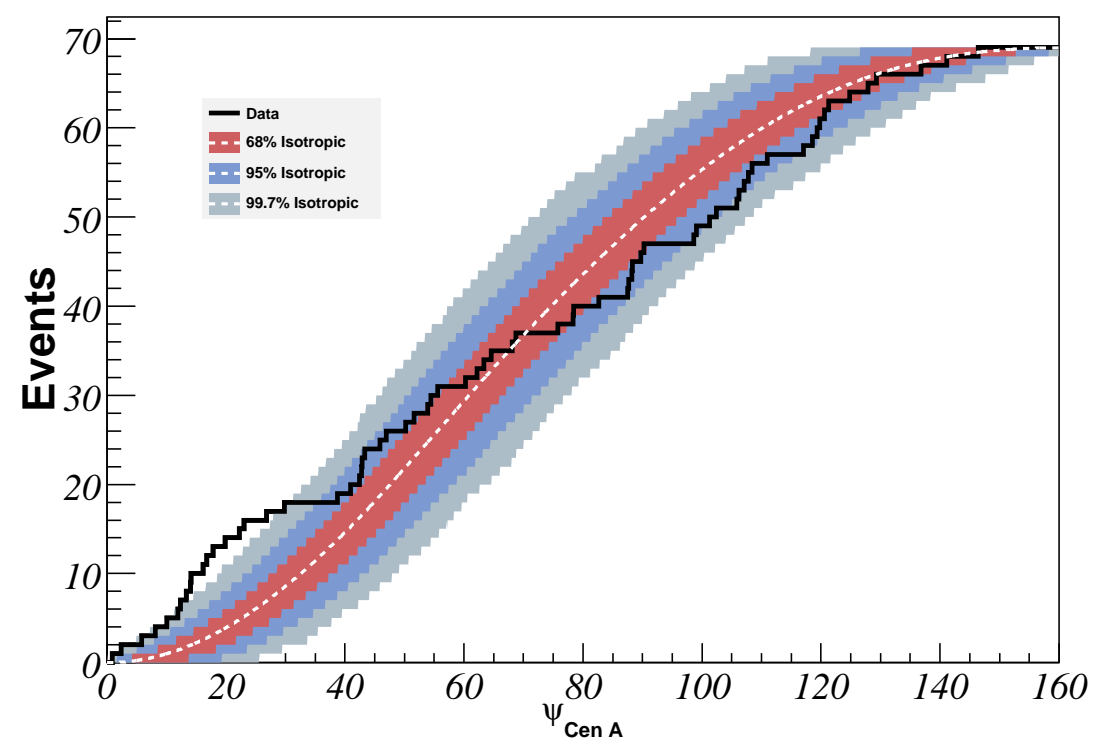

Figure 7: Cumulative number of events with $E \geq 55 \mathrm{EeV}$ as a function of angular distance from the direction of Cen A. The bands correspond to the $68 \%, 95 \%$, and $99.7 \%$ dispersion expected for an isotropic flux. [11].

produced in the central source, along the jet, d) acceleration behind the bow shocks at the edge of the jets where they impact supersonically with the local intergalactic medium, among others. It is quite probable that all these processes are occurring concomitantly. See [18-20] and references within.

In this sense, Cen A is the prototype source and the study of these classes of objects is within the scope of the next years of operation of the Pierre Auger Observatory. The questions presented above and many other important problems are yet to be solved so that we can draw a coherent picture of the high energy cosmic ray scenario. The answers can only be expected from the increasing number of events measured by the Pierre Auger together with innovative data analysis.

\section{References}

[1] The Pierre Auger Collaboration. Properties and performance of the prototype instrument for the Pierre Auger Observatory. Nuclear Instruments and Methods in Physics Research A, 523:50-95, 2004.

[2] The Pierre Auger Collaboration. Correlation of the highest energy cosmic rays with nearby extragalactic objects. Science, 318:938-943, 2007.

[3] The Pierre Auger Collaboration. Measurement of the depth of maximum of extensive air showers above $10^{18}$ eV. Physical Review Letters, 104, 2010.

[4] The Pierre Auger Collaboration. Observation of the suppression of the flux of cosmic rays above 4x10 ${ }^{19}$ eV. Phys.Rev.Lett., 101:061101, June 2008.

[5] John Linsley. Evidence for a primary cosmic-ray particle with energy $10^{20} \mathrm{eV}$. Phys. Rev. Lett., 10(4):146-148, Feb 1963. 
[6] Kenneth Greisen. End to the cosmic-ray spectrum? Phys. Rev. Lett., 16(17):748-, April 1966.

[7] G. T. Zatsepin and V. A. Kuz'min. ZhETF Pis'ma, 4:114, 1966.

[8] N. Hayashida, K. Honda, M. Honda, S. Imaizumi, N. Inoue, K. Kadota, F. Kakimoto, K. Kamata, S. Kawaguchi, N. Kawasumi, Y. Matsubara, K. Murakami, M. Nagano, H. Ohoka, M. Takeda, M. Teshima, I. Tsushima, S. Yoshida, and H. Yoshii. Observation of a very energetic cosmic ray well beyond the predicted $2.7 \mathrm{~K}$ cutoff in the primary energy spectrum. Phys. Rev. Lett., 73(26):3491-, December 1994.

[9] The HiRes Collaboration. Monocular measurement of the spectrum of uhe cosmic rays by the FADC detector of the HiRes experiment. Astroparticle Physics, 23(2):157-174, March 2005.

[10] M.-P. Véron-Cetty and P. Véron. A catalogue of quasars and active nuclei: 12th edition. Astronomy and Astrophysics, 455(2):773-777, aug 2006.

[11] The Pierre Auger Collaboration. Update on the correlation of the highest energy cosmic rays with nearby extragalactic matter. Astroparticle Physics, 34(5):314 - 326, 2010.

[12] The Pierre Auger Collaboration. Correlation of the highest-energy cosmic rays with the positions of nearby active galactic nuclei. Astroparticle Physics, 29(3):188 - 204, 2008.

[13] J. Knapp, D. Heck, S. J. Sciutto, M. T. Dova, and M. Risse. Extensive air shower simulations at the highest energies. Astroparticle Physics, 19(1):77 - 99, 2003.

[14] The Pierre Auger Collaboration. An upper limit to the photon fraction in cosmic rays above $10^{19} \mathrm{eV}$ from the Pierre Auger Observatory. Astroparticle Physics, 27(2-3):155-168, March 2007.

[15] The Pierre Auger Collaboration. Upper limit on the diffuse flux of ultrahigh energy tau neutrinos from the Pierre Auger Observatory. Phys. Rev. Lett., 100(21):211101, May 2008.

[16] R. P. Kraft, W. R. Forman, M. J. Hardcastle, M. Birkinshaw, J. H. Croston, C. Jones, P. E. J. Nulsen, D. M. Worrall, and S. S. Murray. The jet heated x-ray filament in the Centaurus A northern middle radio lobe. The Astrophysical Journal, 698(2):2036, 2009.

[17] D. Whysong and R. Antonucci. Thermal emission as a test for hidden nuclei in nearby radio galaxies. The Astrophysical Journal, 602(1):116, 2004.

[18] The H.E.S.S Collaboration. Discovery of very high energy $\gamma$-ray emission from Centaurus A with H.E.S.S. The Astrophysical Journal Letters, 695(1):L40, 2009.

[19] de Gouveia Dal Pino, E. M., Piovezan, P. P., and Kadowaki, L. H. S. The role of magnetic reconnection on jet/accretion disk systems. $A \& A, 518$ :A5, 2010.

[20] Gopal-Krishna, Peter L. Biermann, Vitor de Souza, and Paul J. Wiita. Ultra-high-energy cosmic rays from Centaurus A: Jet interaction with gaseous shells. The Astrophysical Journal Letters, 720(2):L155, 2010. 